\title{
Eine Dekade nach der Vereinigung: Auf dem Weg zur inneren Einheit?
}

Eine Dekade nach der Wende ist das Bedürfnis nach einer Bestandsaufnahme groß. Was sind die Ergebnisse der Transformationsprozesse in Ostdeutschland? Mit der Vereinigung der beiden deutschen Staaten erfolgte ein völliger Austausch von Institutionen in Ostdeutschland bei gleichzeitiger Übernahme westdeutscher Institutionen. Die damit verbundenen Transformationsprozesse sind zwar auf formaler Ebene abgeschlossen, ihre Wirkungen reichen jedoch weiter und beeinflussen Wirtschaft, Gesellschaft und Politik der gesamten Bundesrepublik Deutschland bis heute. Nun werden Transformationsprozesse nicht nur auf der Ebene von Institutionen vollzogen. Vielmehr sind es Menschen, die in diesen Institutionen handeln und die durch ihre Einstellung und ihr Verhalten die Art der Anpassungen an die Transformation und die Folgen für den weiteren sozialen Wandel beeinflussen.

Die Vereinigung der beiden deutschen Staaten nach vier Dekaden politischen und sozio-ökonomischen Eigenlebens erforderte Anpassungen, auf die die Bevölkerung nicht vorbereitet war. Diese Anpassungen sollten dem Ziel einer institutionellen Vereinigung und inneren Einheit dienen. Mit innerer Einheit ist häufig eine Angleichung zwischen Ost- und Westdeutschen gemeint, und zwar einerseits in den objektiven Lebensbedingungen und andererseits in individuellen Werthaltungen und subjektiver Lebenszufriedenheit. Dieser Beitrag diskutiert, ob diese Kriterien ausreichend sind. Hier wird erörtert, ob innere Einheit vielmehr erst entwickelt ist, wenn eine gegenseitige positive Wertschätzung auf der Grundlage einer primär auf Gemeinsamkeit ausgerichteten Selbstwahrnehmung und sozialen Identität besteht. Daher ist die Kenntnis der individuellen Orientierungen in beiden Landesteilen von Bedeutung. Hier liegen Bedingungen für politisches Verhalten, für die Qualität von Beziehungen zwischen der ost- und westdeutschen Bevölkerung, für die Bereitschaft zur Verantwortung für gemeinsame Problemlösungen und Entscheidungen und für eine gemeinsame soziale Identität. 


\section{Innere Einheit als psychologisches Problem}

\subsection{Aspekte von innerer Einheit}

In dem vorliegenden Band wird in verschiedenen Beiträgen die Frage behandelt, ob trotz der Vereinigung auf institutioneller Ebene eine psychologische innere Mauer besteht, die eine Vereinigung im Sinne einer psychologischen Einheit oder gemeinsamen sozialen Identität blockiert. Dafür wird untersucht, welche individuellen und kollektiven Erfahrungen Menschen in Ostdeutschland nach der Wende gemacht haben, wie diese Erfahrungen verarbeitet wurden und ob damit der Proze $B$ der Entwicklung einer inneren Einheit eher gefördert oder verhindert wurde. Wenn die psychologischen Probleme, die mit den Transformationsprozessen für die ostdeutsche Bevölkerung verbunden gewesen sind - Bedrohung der Identität, Erleben von Unsicherheit, Anpassungsanforderungen an neue Kontexte - inzwischen erfolgreich überwunden sind, wären Voraussetzungen für eine innere Einheit gegeben. Worin läßt sich innere Einheit erkennen?

Vielfach wird bereits die Akzeptanz des Grundgesetzes als Grundlage der nationalen Identität gesehen (vgl. Lepsius, 1995). Besteht jedoch bereits eine innere Einheit, wenn die Bevölkerung der neuen und alten Bundesländer wie dies der Fall ist - die gemeinsamen Grundlagen der staatlichen Ordnung akzeptiert?

Des weiteren wird häufig in einer Angleichung objektiver Lebensbedingungen eine Voraussetzung für innere Einheit gesehen, wobei unberücksichtigt bleibt, daß in ökonomischer und sozialer Hinsicht heute erhebliche Unterschiede innerhalb der neuen Länder bestehen (vgl. Winkler, in diesem Band). Eine globale Gegenüberstellung von neuen und alten Bundesländern ohne Berücksichtigung von jeweiligen demographischen und regionalen Differenzen greift zu kurz (vgl. Nauck \& Joos, 1996). Unzureichend ist auch ein schlichter Vergleich ökonomischer Indikatoren ohne Berücksichtigung der subjektiven Bewertung (Trommsdorff, in Druck a) und der ihnen zugrundeliegenden Werte. Daher wird häufig auch in einer Angleichung oder Ähnlichkeit von Werten ein Indikator für innere Einheit gesehen. Allerdings bestehen unterschiedliche Auffassungen darüber, ob eine Angleichung von Werthaltungen der ost- und westdeutschen Bevölkerung erfolgt ist oder ob z. B. im Bereich politischer, wirtschafts- oder familienbezogener Werte unterschiedliche Einstellungen fortbestehen, sich neu entwickelt oder verstärkt haben (Meulemann, 1996, 1998; Trommsdorff, in Druck a, b).

Auch wenn sich Werte, Einstellungen und Verhaltensmuster angeglichen haben - die Ähnlichkeit (oder Verschiedenheit) von Werthaltungen und Verhalten ist kein ausreichendes Kriterium, um zu beurteilen, ob und wieweit eine psychologische Einigung erfolgt ist. Zum einen liegt das an einer Reihe 
von methodischen und theoretischen Problemen, die sich vor allem auf die Validität und funktionale Äquivalenz von Indikatoren zur Messung von Ähnlichkeit in Werthaltungen beziehen (vgl. Trommsdorff, in Druck a). Zum anderen muß auch ein valider, reliabler und objektiver Nachweis von Ähnlichkeiten (z. B. in Einstellungen) keineswegs bedeuten, daß sich die Personen gegenseitig als ähnlich wahrnehmen. So haben Schmitt und Janetzko (1994) belegt, daß die gegenseitige Wahrnehmung der Ost- und Westdeutschen nicht symmetrisch ist. Des weiteren beruht die soziale Identität von Ost- und Westdeutschen auf unterschiedlichen Kategorien der gegenseitigen Wahrnehmung und Beurteilung (Kessler, Mummendey \& Klink, in diesem Band).

Die in der subjektiven Wahrnehmung und Bewertung wirksamen sozialen Kategorien „Ost versus West" verhindern eine gemeinsame Gruppenzugehörigkeit, die als eine Voraussetzung für eine innere Einheit gesehen werden kann. Vielmehr fördert die soziale Kategorisierung die Akzentuierung von Eigen- und Fremdgruppenidentität mit den damit verbundenen Diskriminierungstendenzen sowie sozialen und politischen Folgeproblemen. Eine gegenseitig wahrgenommene Ähnlichkeit und darüber hinaus eine gegenseitige "Grundsympathie" sind relevant für die Qualität der sozialen Beziehung. Mit der subjektiv gegenseitig wahrgenommenen Ähnlichkeit und Verschiedenheit hängen interpersonale Beziehungen wie Attraktivität und Sympathie, interpersonales Verhalten wie Kooperation und die subjektive Bewertung der eigenen Person als Teil einer sozialen Gruppe sowie die Bereitschaft zur Identifikation mit dieser Gruppe zusammen. Dies ist darum so bedeutsam, weil die Identifikation mit einer sozialen Gruppe gleichzeitig Teil der sozialen Identität ist.

Zusammenfassend heißt dies: Auch wenn teilweise Übereinstimmungen zwischen der ost- und westdeutschen Bevölkerung in bezug auf Werthaltungen und Verhalten berichtet werden (vgl. Hormuth et al., 1996; Gensicke, 1996), so muß dies noch nicht bedeuten, daß eine innere Einheit erfolgt ist, vor allem, wenn man diese als Teil von gemeinsamer sozialer Identităt versteht. Aus psychologischer Sicht sind Akzeptanz der Verfassung und Werteähnlichkeit auch darum keine hinreichende Kriterien für eine innere Einheit, weil damit nicht die Qualität von Bindung an ein neues System und die gegenseitige Wahrnehmung und Akzeptanz aus ost- sowie aus westdeutscher Perspektive erfaßt wird. Unter innerer Einheit werden hier daher nicht nur geteilte Werthaltungen und Ziele verstanden, sondern darüber hinaus gegenseitige Akzeptanz und eine ,geteilte" soziale Identität.

Ist also fast eine Dekade nach der Vereinigung und mit der politischen Herstellung der deutschen Einheit eine psychologische Einheit im Sinne einer gemeinsamen sozialen Identität von Ost- und Westdeutschen erreicht worden? 


\subsection{Innere Einheit als Ergebnis von Akkulturationsprozessen}

Welche psychologischen Prozesse sind erforderlich, um eine innere Einheit in dem oben skizzierten Sinn zwischen ost- und westdeutscher Bevölkerung zu erreichen? Die Qualität der inneren Einheit in bezug auf geteilte Werte, gegenseitige Akzeptanz und gemeinsame Identität kann ein Ergebnis von gemeinsamen Sozialisationserfahrungen und Kulturkontakten sein. Wenn man davon ausgeht, daB in 40 Jahren unterschiedlicher sozio-politischer Kontexte die ost- und westdeutsche Bevölkerung unterschiedlich sozialisiert wurde, müßten damit unterschiedliche Werthaltungen und Verhaltenspräferenzen entstanden sein, die unterschiedliche Ziele und Bindungen an unterschiedliche „Kulturen" bedeuten.

Mit der politischen Vereinigung der beiden deutschen Staaten wurde der vor der Wende nur mühsam mögliche „Kultur-Kontakt" endlich realisierbar. Nach der Typologie von Berry (1990) vermittelt jeder Kulturkontakt den beteiligten Personen in jeder der beteiligten Kulturen eine Vielfalt von Handlungsalternativen. Daraus entwickeln sich über den Zeitverlauf schließlich Akkulturationsstrategien mit relativ stabilen Verhaltensmustern bei unterschiedlichen möglichen Merkmalen: Integration, Assimilation, Segregation und Marginalisierung (Berry \& Kim, 1988). Mit diesen verschiedenen Akkulturationsergebnissen sind unterschiedliche Anpassungsformen an die andere Kultur aufgrund von eigener kultureller Identität einerseits und Interaktionspräferenz mit den Angehörigen der anderen Kultur oder Gruppe andererseits verbunden. Bei Integration wird an den Besonderheiten der eigenen Kultur festgehalten, wobei gleichzeitig Kontakte mit Angehörigen der anderen Kultur erfolgen. Damit ist eine Zugehörigkeit zur bisherigen (Minoritäts-) sowie zur Majoritätskultur gegeben. Integrationserfolge sind besonders bei Jugendlichen bedeutsam, die aufgrund ihrer entwicklungspsychologisch besonderen Lage der Identitätsfindung und Wertebildung im Übergang in das Erwachsenenalter eine doppelte Anpassungsaufgabe zu bewältigen haben. Bei Assimilation werden die Besonderheiten der bisherigen Kultur aufgegeben und die Werthaltungen und Verhaltensbesonderheiten der aufnehmenden Kultur übernommen. Bei Festhalten an der eigenen Kultur und gleichzeitigen Einschränkung des Kontaktes mit der aufnehmenden Kultur führt dies zur Segregation. Schließlich ist Marginalisierung zu erwarten, wenn man weder an den Besonderheiten der eigenen Kultur festhält, noch bereit ist, Kontakte mit der aufnehmenden Kultur einzugehen.

Diese vier Strategien legen unterschiedliche Flexibilität und Anpassungsbereitschaft sowie ganz unterschiedliche Bindungen an bisherige Werthaltungen auf Seiten der Handelnden sowie unterschiedliche Handlungsoptionen und Restriktionen auf Seiten der ,aufnehmenden" Kultur voraus. Vermutlich sind sowohl die individuelle Handlungsorientierung mit den unterschiedlichen in der bisherigen Biographie aufgebauten Problemlösekompetenzen als auch 
die Handlungsoptionen sowie vor allem deren subjektive Wahrnehmung und Bewertung eine zentrale Voraussetzung für die Art der Akkulturationsergebnisse.

Welche Entwicklungen - Assimilation, Segregation, Integration oder Marginalisierung - sind gemäß der Akkulturationstheorie (vgl. Berry, 1990) für die ostdeutsche Bevölkerung im Kontext der westdeutschen Kultur zu erwarten? Wird die Anpassung der ostdeutschen Bevölkerung an Werthaltungen und Verhaltenspräferenzen der westdeutschen Bevölkerung im Sinne einer Assimilation der Minorität an die Majorität der normale Endzustand dieses Akkulturationsprozesses sein?

Dies ist nicht selbstverständlich. Zum einen ist durchaus anzunehmen, daß Minoritätseinflüsse wirksam sein können in der Weise, daß Werthaltungen und Verhaltensmuster der westdeutschen Bevölkerung durch konsistente und wiederholte Verhaltensweisen der ostdeutschen Bevölkerung verändert werden können. Solche Einflüsse von Minoritäten sind seit Moscovicis (1985) Untersuchungen zum sozialen Wandel bekannt. Da hier komplizierte Wechselwirkungsprozesse zwischen dem handelnden Individuum und seinem sozialen Kontext (sowohl dem bisherigen wie dem neuen) anzunehmen sind, ist jedenfalls eine Vorhersage der Akkulturationsergebnisse schwierig. Des weiteren wird die Vorhersage durch den Zeitfaktor erschwert, und zwar auf verschiedene Weise: Zum einen ist von Bedeutung, welches Entwicklungsalter die handelnden Personen der Minoritätskultur haben, z. B. ob sie sich selbst noch im Stadium der Identitätsbildung im Jugendalter mit verschiedenen möglichen Identitätsformen auseinandersetzen, und welche Entwicklungsaufgaben sie zum gegebenen Zeitpunkt zu erfüllen haben. Zum anderen ist von Bedeutung, über welche Zeit und mit welchen Erwartungen (und mit welchem bisherigen Ergebnis) der ProzeB der Kulturbegegnung verläuft. Dabei sind sowohl die eigenen Erwartungen als auch die des weiteren sozialen Umfeldes relevant.

Wenn man also Akkulturation als einen über den Zeitverlauf erfolgenden Prozeß versteht, so können über die Zeit hinweg ganz verschiedene Anpassungsmuster auftreten. Anfängliche Akzeptanz und Euphorie für westdeutsche Werthaltungen und Verhaltensmuster können einer späteren Distanzierung Platz machen. Anfängliche Euphorie bei Begegnung mit anfangs hoch geschätzten Lebensstilen kann einer Ernüchterung oder Enttäuschung weichen. Solche Verläufe im Sinne einer u-kurvenförmigen Entwicklung bei Kulturkontakten sind möglich und empirisch nachgewiesen.

Auf der einen Seite lassen sich empirische Daten zu Sozialisations- und Anpassungsergebnissen nach der Wende in Ostdeutschland im letzten Jahrzehnt aus akkulturationstheoretischer Sicht als eine erfolgreiche Integration interpretieren. Auf der anderen Seite legen Beiträge des vorliegenden Bandes signifikante Segregationstendenzen nahe. So zeigen sich Unzufriedenheiten bzw. reduziertes Wohlbefinden sowohl bei der ost- als auch der westdeut- 
schen Bevölkerung. Die anfangs eher positiven und optimistischen Bewertungen der Lebenslage durch die Bewohner der neuen Länder haben sich im Laufe der Transformation verschlechtert (Bierhoff; Montada \& Dieter; Winkler; in diesem Band).

\section{Lebenszufriedenheit}

Die subjektive Bewertung der eigenen Lebenslage kann als eine Grundlage für soziale Integration und Identifikation mit der sozialen Gruppe und dem gegebenen System und damit für die Qualität der inneren Einheit gesehen werden. Daher soll im folgenden der Frage nach den Bedingungen für Lebenszufriedenheit und Wohlbefinden nachgegangen werden.

\subsection{Bewertungskriterien}

Worauf beruht subjektiv erlebte Unzufriedenheit in Ostdeutschland, wenn seit der Wende zumindest in ökonomischer Hinsicht massive Gewinne erzielt wurden? Wirtschaftliche und andere objektivierbare "Gewinne" werden offenbar subjektiv verschieden bewertet, z. B. im Vergleich zu wahrgenommenen Kosten, Investitionen und Verlusten, wie dem Verlust an Arbeitssicherheit und sozialer Versorgung. Objektivierbare Gewinne und Verluste sind psychologisch wirksam durch deren subjektive Wahrnehmung und Bewertung, die auf Vergleichen beruht. Je nachdem, welchen Vergleichsstandard man wählt - einen temporalen intraindividuellen, auf vergangene Erfahrungen und subjektive Wünsche und Erwartungen bezogenen, oder einen interindividuellen, auf soziale Vergleiche mit ähnlichen oder unähnlichen Personen oder sozialen Gruppen bezogenen Standard - , können die Gewinne relativ unterund die Verluste überbewertet werden. Lebenszufriedenheit und Wohlbefinden werden hier als Ergebnis der subjektiv nach bestimmten Kriterien bewerteten Lebenslage definiert. Die Bewertung der Lebenslage kann durch Vergleiche mit der eigenen Vergangenheit und den Erwartungen für die Zukunft (temporaler Vergleich) sowie durch Vergleiche mit anderen Personen oder Gruppen (sozialer Vergleich) erfolgen.

Abnehmendes Wohlbefinden könnte einerseits ein Ergebnis der Verschiebung von Kriterien von anfangs temporalen zu später sozialen Vergleichen (mit der westdeutschen Bevölkerung) oder andererseits schlicht ein Ergebnis von enttäuschten Erwartungen sein. Nach der Wende bestand die allgemeine Überzeugung, daß die Transformation schnell und erfolgreich gelingen und der Lebensstandard der ost- und westdeutschen Bevölkerung sich schnell angleichen würde. Die ökonomischen Bedingungen in Ost- und Westdeutsch- 
land unterscheiden sich heute jedoch immer noch, auch wenn sich die ökonomischen und sozialen Bedingungen in den neuen Ländern im Vergleich zur Zeit vor der Wende insgesamt ganz erheblich verbessert haben.

Montada und Dieter (in diesem Band) belegen, daß die ostdeutsche Bevölkerung sowohl für private wie für öffentliche Lebensbereiche die Lage nach der Wende im Vergleich zu vorher als negativer einschätzt. Damit stellt sich die Frage, warum sich objektiv gegebene Verbesserungen ( $\mathrm{z}$. B. in Bezug auf politische und persönliche Freiheit) nicht in entsprechenden subjektiven Wahrnehmungen und Bewertungen spiegeln bzw. warum gegenüber objektiven Gewinnen jedoch subjektiv erlebte Verluste überwiegen. Darüber hinaus kommt die west- und ostdeutsche Bevölkerung bei der Bewertung der Gewinne und Verluste der jeweils anderen Gruppe und bei der Beurteilung der Ursachen dafür jeweils zu unterschiedlichen Urteilen. Das ist zum einen erklärungsbedürftig und fordert zum anderen Fragen nach den Wirkungen solcher Einschätzungen für den Prozeß der psychologischen Einigung heraus. Diese Bewertungsergebnisse sollten Folgen haben für das subjektive Wohlbefinden und damit für die verschiedensten Bereiche des sozialen Handelns - für Konfliktbereitschaft und Kooperation, Mobilität, politisches Verhalten. Schon daher ist es ein wichtiges Unterfangen, Bedingungen des psychischen Wohlbefindens und der Zufriedenheit der ost- und westdeutschen Bevölkerung zu untersuchen. $\mathrm{Zu}$ diesem Fragenkomplex bestehen verschiedene theoretische $\mathrm{Zu}$ gänge. Ein besonders aufschlußreicher Zugang wird in diesem Band durch Untersuchungen zum Gerechtigkeitserleben vermittelt.

\subsection{Gerechtigkeitserleben als Voraussetzung für Zufriedenheit}

Wenn Gerechtigkeitsstandards verletzt werden, wirkt sich dies nach der Theorie von Montada $(1994,1997)$ negativ auf das subjektive Wohlbefinden aus. Wenn man von einem Bedürfnis nach Gerechtigkeit ausgeht, ist zu erwarten, daß erlebte Ungerechtigkeit das Bedürfnis aktiviert, Gerechtigkeit wiederherzustellen. Mit erlebter Ungerechtigkeit sind Erfahrungen von Kränkung und Selbstwertverletzung verbunden. Dem Erleben von Ungerechtigkeit liegt die wahrgenommene Diskrepanz zwischen erwarteten ,gerechten"und tatsächlich erlebten Belohnungen zugrunde. Diese Diskrepanzwahrnehmung ist jedoch keineswegs durch "cool cognitions" gekennzeichnet, sondern mit mehr oder weniger unangenehmen Emotionen verbunden. Je nachdem, welche Ursache für diese Diskrepanz verantwortlich gemacht wird, welchen Lebens- und Wertebereich diese Diskrepanz betrifft und welche Hoffnungen bestehen, diese Diskrepanz zu überwinden, können Emotionen wie Ärger, Neid, Enttäuschung, Schuld aktiviert werden.

So können sowohl positive Abweichungen von erwarteter gerechter Verteilung im Sinne von wahrgenommener ungerechtfertigter Verdienste oder 
Bereicherung (relative Privilegierung) als auch negative Abweichungen im Sinne wahrgenommener ungerechtfertigter Schlechterstellung (relative Deprivation) unterschiedliche negative Emotionen induzieren und ein Bedürfnis nach Wiederherstellung von Gerechtigkeit aktivieren. Dieses Bedürfnis kann wiederum ganz unterschiedliche Verhaltensweisen aktivieren, die dem Ziele dienen, das Diskrepanzerleben und die negativen Emotionen zu reduzieren. Negative Emotionen lassen sich $u$. a. durch Abwertung der Quelle der Ungerechtigkeit reduzieren, was die Beziehung zwischen den Akteuren nicht verbessert. Dabei können auch solche Verhaltensweisen auftreten, die als sozial unerwünscht oder unangepaßt gelten, wie z. B. Aggression, Protest oder Apathie.

Die Ergebnisse von Schmitt, Maes und Schmal (in diesem Band) belegen, daß erlebte Ungerechtigkeiten in bezug auf die Verteilung von Lebensqualität im Bereich von Arbeit und Beruf in Ost- und Westdeutschland mit emotionalen Belastungen und beeinträchtigter psychischer Gesundheit verbunden sind. Je nach Deutung der Verteilung von Ressourcen in Ost- und Westdeutschland werden Gerechtigkeitsbewertungen aktiviert, die im Fall von Ungerechtigkeitserleben negative Emotionen evozieren, welche das weitere Handeln lenken. Im Fall von ungerechtfertigt erlebtem eigenen Vorteil erhöht sich die Bereitschaft, auf Vorteile zu verzichten oder prosozial zu handeln. Im Fall von ungerechtfertigt erlebten Nachteilen erhöht sich die Bereitschaft zur Auflehnung oder Segregation. Diese Befunde können aus der Theorie der relativen Deprivation und der Theorie der relativen Privilegierung abgeleitet werden.

$\mathrm{DaB}$ es allerdings nicht quasi automatisch zu Ungerechtigkeitserleben kommen muß, wenn man selbst schlechter als andere gestellt ist, belegen die Ergebnisse von Bierhoff (in diesem Band). Danach waren Ostdeutsche kurz nach der Wende nicht nur ausgesprochen zuversichtlich in bezug auf ihre zukünftige Lebenslage, sie beurteilten auch ihre objektiv gegebene Schlechterstellung (z. B. im Bereich von Beruf, Einkommen, Bildung, Wohnung, Konsum) als ähnlich gut im Vergleich zu Westdeutschen. Ostdeutsche beurteilten die erlebten Veränderungen zu Beginn der Transformation als relativ positiv; sie erlebten auch objektive Einschränkungen und Unsicherheit (besonders im Berufsbereich) eher nicht als beeinträchtigend. Zur Zeit nach der Wende beurteilten Ostdeutsche offenbar ihre Lebenslage zunächst vorwiegend aufgrund temporaler Vergleiche; sie verankerten also Vergleiche in der Zeit vor der Wende. Im Laufe des sozialen Wandels und der Transformation nach der Wende bevorzugten Ostdeutsche dann zunehmend mehr soziale Vergleiche, vermutlich aufgrund der Erwartung einer schnellen erfolgreichen Gleichstellung mit dem Westen und mit dem Ergebnis, daß die Beurteilung der eigenen Lebenslage zunehmend negativer wurde. Im Akkulturationsprozeß erfahrene Erwartungsenttäuschungen reduzierten die Integrationsbereitschaft.

In soziale Vergleiche gehen offenbar in besonderem Maße Gerechtigkeitsvorstellungen mit ein. Dies ist plausibel, weil Kriterien, nach denen die 
eigene soziale Lage beurteilt wird, Vorstellungen über die eigene Bedürftigkeit (und die von anderen Personen) sowie über gerechte Verteilung von Ressourcen beinhalten. Damit können Erwartungen induziert werden, wie die eigene Lebenslage im Vergleich zu anderen gerechterweise eigentlich aussehen müßte. Für die ostdeutsche Bevölkerung erbrachten soziale Vergleichsurteile und darauf beruhende Bewertungen eine negative Bilanz. Dies aktivierte vermutlich erneut temporale Vergleiche, die die Vergangenheit in einem besseren Licht erscheinen lassen als die gegenwärtige Lebenslage.

\subsection{Werthaltungen als Grundlage für Zufriedenheit}

Das Bewertungsergebnis hängt ab von eigenen Werthaltungen, Wünschen und Zielen. Lange Zeit wurden Ähnlichkeiten von Werthaltungen bei Ostund Westdeutschen als Kriterium für eine innere Einheit verwendet, ohne zu berücksichtigen, daß formal ähnliche Werthaltungen noch nicht unbedingt gegenseitige Akzeptanz und Integration implizieren. So können Gerechtigkeitswerte bei der ost- und westdeutschen Bevölkerung ähnlich sein, aber die Bewertung der eigenen Lebenslage kann sich bei Anwendung solcher Gerechtigkeitswerte erheblich unterscheiden und eine ganz unterschiedliche Lebenszufriedenheit bewirken.

Des weiteren ist aus motivationstheoretischer Sicht für die Vorhersage von subjektiver Zufriedenheit die subjektive Wahrnehmung relevant, wie gut eigene Werthaltungen realisiert werden - in der Vergangenheit, der Gegenwart und der Zukunft. In diese Wahrnehmung gehen bisherige Erfahrungen, die Zukunftsorientierung (Trommsdorff, 1994) und Kontrollüberzeugungen eigener Wirksamkeit (self efficacy; Bandura, 1997) ein. Die Lebenszufriedenheit müßte daher mit der Bedeutung der jeweiligen Werthaltungen im individuellen Wertesystem und der erlebten und erwarteten Realisierungschancen zusammenhängen.

Wenn man davon ausgeht, daB interindividuelle Unterschiede in Zielen, Werten und damit der Wichtigkeit von Ereignissen bestehen, z. B. in bezug auf verschiedene Berufs- oder Freizeittätigkeiten, müßte die Erfüllung bzw. Nichterfüllung von individuellen Zielen zu unterschiedlicher Ausprägung von Zufriedenheit führen. Wenn also Berufstätigkeit - wie für die Bevölkerung in den neuen Ländern üblicherweise der Fall - ein wichtiger Wert ist, müBte Arbeitslosigkeit in hohem Maße frustrierend sein. Daher fühlen sich ostdeutsche Frauen, die von Arbeitslosigkeit in hohem Maße betroffen sind, besonders gravierend in ihrer Lebenszufriedenheit beeinträchtigt. Stromberg und Boehnke (in diesem Band) prüfen die Frage, ob die Beeinträchtigung von wichtigen Werthaltungen die subjektive Lebenszufriedenheit reduziert, an einer Stichprobe von ost- und westdeutschen Studierenden. Ostdeutsche Studierende, die eine Verminderung der Realisierbarkeit von für sie wichtigen Traditions-, 
Konformitäts- und Sicherheitswerten im neuen System erlebt haben, berichten über weniger Lebenszufriedenheit. Wenn wichtige Werte nicht bzw. nicht mehr im Einklang mit den gegebenen Bedingungen stehen, also wenn sich die Bedingungen so verändert haben, $\mathrm{da} \beta$ sie diskrepant zu den Werten werden, erwächst Unzufriedenheit. Diese Befunde lassen sich also motivationstheoretisch erklären, wobei Wertepräferenzen als generalisierte Zielorientierungen verstanden werden, auf die hin Menschen ihr Handeln organisieren. Die über einen längeren Zeitraum hinweg wahrgenommene Blockierung der Zielerreichung müßte erhebliche Diskrepanzerlebnisse und Unwohlsein auslösen.

\section{Individuelle und soziale Folgen von Unzufriedenheit}

\section{I Individuelle Verarbeitung von Diskrepanzerlebnissen: Kontrollorientierung}

Da sich individuelle Werthaltungen im SozialisationsprozeB in Zusammenhang mit den Werten der Gruppe, der Gesellschaft und Kultur herausbilden, ist die Realisierbarkeit von Werten relevant für die soziale Identität. Daher wird erlebte Diskrepanz von eigenen bzw. kollektiv geteilten Werthaltungen auf der einen Seite und deren Realisierbarkeit auf der anderen Seite über Unwohlsein hinaus auch identitätsrelevante Folgen haben, insofern als dies als kollektive Identitätsbedrohung erlebt werden kann. Hier kann ein Handlungspotential aufgebaut werden, das anomische oder antisoziale Reaktionen zur Folge hat und Segregation oder Marginalisierung fördert.

Kessler, Mummendey und Klink (in diesem Band) untersuchen, mit welchen Strategien erlebte negative soziale Identität bewältigt wird. Ein Vergleich der Theorie der relativen Deprivation und der Theorie der sozialen Identität geht anhand der empirischen Befunde zugunsten der Theorie der sozialen Identităt aus. Theorien der sozialen Identität und der relativen Deprivation sagen Bewältigungsstrategien vorher, die nicht zuletzt politische Folgen und damit Folgen für sozialen Wandel haben.

Wie werden objektive Verschlechterungen der Lebenslage verarbeitet? Diewald (in diesem Band) belegt, daß der Arbeitsmarkt in den neuen Ländern kaum Chancen einer Aufwärtsmobilität eröffnet hat. Vielmehr sind berufliche Wechsel mit beruflichem Abstieg verbunden, und horizontale Mobilität erfolgt unfreiwillig. Die objektiven beruflichen Verschlechterungen spiegeln sich in der Tat im subjektiven Erleben reduzierter Kontrollierbarkeit und in geringerem subjektiven Wohlbefinden.

Beruht die subjektive Deutung der Lebenslage auf Gerechtigkeitsurteilen, nach denen die eigene Person oder die eigene Gruppe als Opfer ungerechten 
Handelns wahrgenommen wird, sollten Problemlösestrategien mit Zeitperspektive, Verzicht-, Anstrengungs- und Aufschubbereitschaft weniger gewählt werden. Vielmehr ist es wahrscheinlich, daß der einzelne auf die erlebten Enttäuschungen wie Arbeitslosigkeit frustriert reagiert und durch Rückzug oder Verweigerung die eigene Lage verschlimmert. Allerdings können soziale und individuelle Ressourcen das Ergebnis von Anpassungs- und Bewältigungsverhalten beeinflussen (Trommsdorff, in Druck b, c). Zu den individuellen Ressourcen gehören Persönlichkeitsmerkmale wie die Bereitschaft, Veränderungen zu akzeptieren (vgl. Stromberg \& Boehnke, in diesem Band). Zu den sozialen Ressourcen gehört z. B. die Akzeptanz durch andere Personen oder Gruppen, was u. a. durch soziale Netze am Arbeitsplatz erfolgen kann (vgl. Hahn, in diesem Band).

Die Untersuchungen zu Bedingungen und Folgen von Arbeitslosigkeit von Hahn (in diesem Band) belegen Wechselwirkungen von objektiven und subjektiven Faktoren beim Erleben von Arbeitslosigkeit. Der Erwerbsstatus beeinflußt die Erfahrung von sozialer Integration und Verhaltenstendenzen, die wiederum den Erwerbsstatus beeinflussen können. In Biographien von Arbeitslosen werden Wechselwirkungen zwischen Arbeitslosigkeit und unterschiedlich erfolgreicher Bewältigung aufgrund von Kontrollerleben und sozialer Integration nachgewiesen. Die aufgrund ungünstiger Problemlösestrategien erfolgende eigene Beeinträchtigung des Wiedereinstiegs in das Erwerbsleben belegt die Bedeutung von subjektivem Erleben objektiv schwieriger Lebenslagen und die aktive Rolle der betroffenen Person, eine objektive Verbesserung der eigenen Lebenslage zu erreichen. Dabei sind vor allem Kontrollüberzeugungen relevant.

Nach der Theorie der Kontrollorientierung (vgl. Rothbaum, Weisz \& Snyder, 1982; Trommsdorff, 1994, 1995) können Kontrollüberzeugungen eher akkommodative oder eher assimilative Funktionen haben. Unklar ist jedoch, welche Handlungen im Falle von Ungerechtigkeitserleben erfolgen. Anpassungen können über verschiedene Handlungen erfolgen, und zwar sowohl über nach außen gerichtete Handlungen der Veränderung von Bedingungen als auch über interne Prozesse der Umdeutung. Diskrepanzen in Erwartungen oder Mißerfolgserlebnisse und Unwohlsein können reduziert werden durch Veränderung des Kontextes (z. B. man sucht einen neuen Kontext auf, bspw. verläßt man den bisherigen Wohnort, weil dort weniger Aussicht auf Arbeit besteht) bzw. durch aktive Umgestaltung der Lebensbedingungen (z. B. Weiterbildungsbemühungen; Mitgliedschaft in einer die eigene Zielerreichung unterstützenden Gruppe bzw. politischen Partei). Diese Strategie der Assimilation (,primäre“ Kontrollorientierung) dient dem Ziel, eine bessere Passung von eigenen Zielen und externen Bedingungen zu erreichen. Diskrepanzerfahrungen können jedoch auch reduziert werden durch interne Anpassungsprozesse wie Verzicht auf die bisherigen Ziele oder deren Umstrukturierung, d. h. auch die Veränderung von deren Bedeutung im bisherigen Zielsystem, d. h. durch 
Akkommodation (bzw. „sekundäre" Kontrollorientierung) (vgl. Trommsdorff, 1989, 1994, 1995). Diese Anpassungsstrategien erfordern allerdings u. a. die Überzeugung von eigener Selbstwirksamkeit, von Optimismus sowie eine Distanzierung von einer Opferrolle. Empirische Befunde für Varianten von Kontrollüberzeugungen und deren Funktion für die Bewältigung von Problemen in Abhängigkeit von eher individualistischen versus eher gruppenbezogenen Werthaltungen werden inzwischen in einigen kulturvergleichenden Untersuchungen berichtet (vgl. Trommsdorff, in Druck b, c; Seginer, Trommsdorff \& Essau, 1993; Essau \& Trommsdorff, 1993; Trommsdorff \& Essau, in Druck).

Welche Strategien werden in den neuen Ländern bevorzugt? Werden Veränderungen in der Umwelt, wie z. B. neue Verteilung von Ressourcen, oder interne kognitive Umdeutungen, also eher assimilative oder akkommodative Prozesse der primären oder sekundären Kontrollorientierung bevorzugt? Die Befunde von Kessler, Mummendey und Klink (in diesem Band) zeigen, daß die Bewältigung von negativer sozialer Identität in Ostdeutschland sowohl über den Wechsel von Vergleichsdimensionen bzw. der Verankerung der Vergleiche (Akkommodation) als auch über den Wechsel der Gruppe (Assimilation) erfolgen kann. Die Wahl der einen oder anderen Anpassungsform beeinflußt wohl die subjektive Lebenszufriedenheit weniger als vielmehr der subjektive Erfolg in bezug auf den subjektiv relevanten Vergleichsmaßstab. Beide Strategien sollten jedenfalls Unzufriedenheit reduzieren.

Für Vorhersagen über den Verlauf einer inneren Einigung und des weiteren sozialen Wandels ist jedoch von Bedeutung, Kenntnisse über die Wahl von "primären" oder "sekundären" Kontrollstrategien zu haben. Die Strategie der sekundären Kontrolle könnte z. B. zu einer Angleichung von Werthaltungen von Ost- und Westdeutschen führen. Allerdings ist zu fragen, ob Angleichungen von Werthaltungen, die sich als diskrepant zu den Gegebenheiten erwiesen haben, mit entsprechender innerer Überzeugung so umstrukturiert werden, daß Änderungen innerhalb des internen Wertesystems erfolgen. Anpassungsstrategien an erlebte Wertediskrepanzen durch sekundäre Kontrolle können eine zunehmende Akzentuierung bisheriger Werthaltungen und eine Aufwertung der "Eigengruppe" und damit eine Akzentuierung von eigener sozialer Identität in Abgrenzung von der "Aufnahmekultur" bewirken. Die Befunde von Montada und Dieter (in diesem Band) lassen sich in diesem Sinne deuten. Die Autoren zeigen, daß Ostdeutsche die früheren Errungenschaften der damaligen DDR wie Kinderversorgung, Arbeitsplatzsicherheit, Wohnsicherheit etc. insbesondere im Vergleich zu den Gegebenheiten im neuen System vorziehen. Dies deutet auf eine Aufwertung traditioneller kollektiver Werte bei gleichzeitiger Abwertung des gegebenen Systems hin. 


\subsection{Soziale Folgen von Unzufriedenheit}

Gerechtigkeitserlebnisse haben in Zusammenhang mit unserer Frage nach Bedingungen von innerer Einheit insofern eine besondere Bedeutung als sie im Sinne von kollektiven Erfahrungen, vermittelt über die Zugehörigkeit zur ostdeutschen Bevölkerung, wirksam sind. Wenn die eigene Lebenslage als ein mit anderen geteiltes gemeinsames Schicksal ungerechter Verteilung wahrgenommen wird, kann sich das subjektive Erleben in kollektives Erleben transformieren. Damit müßten über individuelle Anpassungsstrategien hinaus Überzeugungen von ,geteilter Kontrolle" (shared control beliefs) sowie damit verbunden auch kollektive Strategien der primären oder sekundären Kontrolle gewählt werden, die politisches Handeln und damit den weiteren sozialen Wandel beeinflussen. Damit stellen sich Fragen, inwieweit Kontrollüberzeugungen als kollektive Überzeugungen (shared control beliefs) eine Funktion der Problembewältigung übernehmen können, die anders als bei auf die eigene Person bezogenen Kontrollüberzeugungen (im Sinne von „self efficacy“, vgl. Bandura, 1997) eine Ausweitung von angenommenen Kontrollmöglichkeiten auf eine ganze Gruppe erlaubt.

Hahn (in diesem Band) weist nach, daß Arbeitslosigkeit nicht als individuell ungerechtes Schicksal, sondern als kollektive Betroffenheit und als gesellschaftliche Ungerechtigkeit erlebt wird. Gleichzeitig werden die Transferleistungen von West nach Ost als gerechtfertigt angesehen. Hier zeigt sich eine spezifische Dimension von Gerechtigkeitserleben im Sinne von kollektiv erfahrenem Schicksal. Dieses Ungerechtigkeitserleben müBte das Bedürfnis nach kollektiver Identität und Kontrolle erhöhen und politische Bewältigungsstrategien fördern, wie sie in nostalgischer Verklärung von historisch gewachsener Gruppenidentität und weiter in politischer Partizipation durch Aktivitäten mit hoher Bindung an Vor-Wende-Erfahrungen möglich sind. Der Zuwachs der PDS-Wähler bestätigt diese Tendenz.

Auch die Befunde von Liebig und Wegener (in diesem Band) zeigen, daß die negative Bewertung eigenen materiellen Erfolges und das kollektive Erleben von Zurücksetzung Ungerechtigkeitserlebnisse aktivieren kann, die politisches Verhalten beeinflussen, allerdings in Ost- und Westdeutschland in unterschiedlicher Weise. Während in Westdeutschland erlebte Ungerechtigkeit eher Protestverhalten aktiviert, reagieren Ostdeutsche eher mit Verweigerung, Rückzug und politischer Apathie. Auch in anderen Studien (z. B. Winkler, in diesem Band) wird eine relativ geringe Bereitschaft von Ostdeutschen zur politischen Aktivität bestätigt. Diese verschiedenen Reaktionen hängen vermutlich mit den in jeweils beiden Teilen Deutschlands sozialisierten Verhaltensmustern zusammen. Politische Demonstrationen galten in der ehemaligen DDR als Ausdruck von Zustimmung gegenüber dem politischen System; im Westen gelten politische Demonstrationen als Ausdruck von Protest. Rückzug 
ins Private war in der alten DDR ein Mittel zur Verweigerung und galt als ein Merkmal der Nischengesellschaft.

Wie sich Ungerechtigkeitserleben auf soziales Verhalten auswirkt, ist jedoch noch nicht geklärt. So ist z. B. zu fragen, ob bei ostdeutschen Frauen ein hohes Potential an Veränderungsbereitschaft der gegenwärtigen Bedingungen in sozio-ökonomischer und politischer Hinsicht besteht, weil sie sich als eigentliche Verlierer erleben, nachdem die Verteilung sozialer Leistungen und die Verteilung an Arbeit zu ihren Ungunsten erfolgt ist. Des weiteren ist zu fragen, ob Frauen diese Benachteiligung als Ungerechtigkeit mit den damit verbundenen negativen Emotionen erleben und ob sie dies als Erfahrungsfilter an ihre Kinder weitergeben. Damit würden Sozialisationsbedingungen der nächsten Generation wenig geeignet sein, eine innere Einigung für die $\mathrm{Zu}$ kunft herbeizuführen.

Bei kollektiv erlebter Unzufriedenheit und Attribuierung der Ursachen für die als negativ wahrgenommene eigene Lebenslage auf die jeweils andere Gruppe ist zu erwarten, daß sich soziale Konflikte verschärfen. Wenn die ostdeutsche die westdeutsche Bevölkerung für die eigene relative Schlechterstellung als verantwortlich sieht und umgekehrt, wenn Westdeutsche eigene erhöhte Verzichtleistungen als politisch erpreßte unverdiente Inanspruchnahme durch Ostdeutsche wahrnehmen, dürfte sich dies jeweils negativ auf die sozialen Beziehungen und eine innere psychologische Einheit auswirken. Soziale Kategorisierungen und negative Stereotype sind die Folge und können auf beiden Seiten Verhalten verstärken, das auf Veränderung der gegebenen Bedingungen zugunsten eigener Besserstellung und zu Lasten der Besserstellung der jeweils anderen Gruppe abzielt. Auf der Grundlage gerechtigkeitstheoretischer Überlegungen ist anzunehmen, daß Intergruppenkonflikte entstehen, wenn eine Gruppe im Vergleich zu einer anderen Gruppe einen Verlust von Gerechtigkeit erlebt und darüber hinaus diese ungerechte Verteilung als Folge des Verhaltens der jeweils anderen Gruppe deutet. Solche Konflikte dürften sich aggravieren, wenn die eine Gruppe sowohl das Ungerechtigkeitserleben als auch die Kausalattribuierung der jeweils anderen Gruppe als unzutreffend wahrnimmt.

Wenn sich also Ostdeutsche als Opfer von ungerechten Verteilungen durch Westdeutsche erleben, und wenn Westdeutsche ihrerseits weder das Ungerechtigkeitserleben der Ostdeutschen noch deren Kausalattribuierung für angemessen halten, sondern im Gegenteil etwaige Schlechterstellungen von Ostdeutschen als Folge von deren eigenem Handeln (bzw. als Folge der politischen und wirtschaftlichen Entwicklung in der ehemaligen DDR oder als Folge mangelnder Umstellungsbereitschaft) deuten, dürfte eine neutrale oder gar positive soziale Beziehung zwischen ost- und westdeutscher Bevölkerung und eine ,innere Einheit" blockiert sein. Ungerechtigkeitserleben kann also Verhaltenskonsequenzen mit Folgen für weiteren sozio-politischen Wandel haben. Die Besonderheit an dem Ansatz von Montada $(1994,1997)$ und Mon- 
tada und Dieter (in diesem Band) für die Analyse des subjektiven Erlebens der Transformation in Ostdeutschland besteht darin, mit dem Ansatz der Theorie der Gerechtigkeit Ursachen für die tiefgreifenden Beschädigungen einer inneren Einheit aufzuzeigen.

\section{Ausblick}

Für einen psychologischen Zugang zur Analyse von sozialem Wandel und der darin wirksamen Prozesse ist eine Analyse von Gerechtigkeitserleben und den damit verbundenen Werthaltungen und Vergleichsprozessen (temporale interne und/oder soziale Vergleiche) sowie deren Veränderungen aufschlußreich, um das subjektive Erleben des sozialen Wandels (einschließlich der Bewertung der eigenen Lebenslage und der Lebenszufriedenheit) und die damit verbundenen Anpassungsprozesse sowie Handlungsfolgen zu erfassen.

Sozialer Wandel ist ein Prozeß mit unterschiedlichem Tempo, mit unterschiedlich ausgeprägten Änderungen und Umbrüchen in verschiedenen Bereichen und zu verschiedenen Zeitpunkten. Daher sind individuelle Verarbeitungen von sozialem Wandel nicht mit der Fokussierung auf nur einen Zeitpunkt angemessen analysierbar. Sozialer Wandel wird nicht zu einem bestimmten Zeitpunkt und schon gar nicht zu Beginn des Einsetzens von sozialem Wandel „bewältigt“. Vielmehr vollzieht sich sozialer Wandel im subjektiven Erleben über die Zeit hinweg, wobei Änderungen der objektiven Umwelt nicht unbedingt im einzelnen registriert und bewertet werden, sondern in ihrem Zusammenspiel mit den jeweils gegebenen individuellen Bedingungen einschließlich der verfügbaren individuellen und sozialen Ressourcen. Darüber hinaus verändern sich im ProzeB des Wandels auch die gewählten Kriterien für die Bewertung der eigenen Lebenslage.

Um die Veränderungen in der subjektiven Fokussierung auf Umweltbedingungen und Veränderungen in der Bewertungsgrundlage solcher subjektiver Wahrnehmungen mit zu berücksichtigen, sind Zeitverlaufsstudien erforderlich. So wäre in zukünftigen Studien die Hypothese zu prüfen, die durch die vorliegenden Befunde nahegelegt wird, nämlich ob zunächst temporale Vergleiche später sozialen Vergleichen Platz machen; und ob im Fall von sozialen Vergleichen eher Gerechtigkeitsvorstellungen wirksam werden, die die Lebenszufriedenheit mindern, wenn sie verletzt werden.

Zur Analyse von Zusammenhängen zwischen sozialem Wandel und individuellem Verhalten sind vor allem theoretische Ansätze und Konzepte relevant, die einerseits erlauben, Wirkungen von sozialem Wandel auf individuelles Handeln und andererseits Wirkungen von individuellem Handeln auf sozi- 
alen Wandel zu untersuchen. Solche angenommenen Wechselwirkungen müßten besonders deutlich werden, wenn man die Transformationsprozesse in Ostdeutschland untersucht.

Die in diesem Band vorliegenden Arbeiten gehören zu den wenigen Studien, die individuelle psychologische Phänomene im sozialen Kontext und darüber hinaus auch im Kontext veränderter sozialer Bedingungen untersuchen. Die Transformation in Ostdeutschland stellt enorme Herausforderungen an die Sozialwissenschaften. Der vorliegende Band zeigt, daB es sich lohnt, sich diesen Herausforderungen zu stellen, und er zeigt, daß es erfolgversprechend ist, solche Analysen auf der Grundlage von sozialwissenschaftlichen Theorien und sophistizierten Methoden vorzunehmen. Die Fruchtbarkeit, aber auch die Grenzen gerechtigkeitstheoretischer Ansätze sind hier deutlich geworden sowie die Notwendigkeit, Erkenntnisse aus den Nachbardisziplinen mit einzubeziehen. Insofern stimmen die hier vorliegenden Arbeiten optimistisch, daß die sozialwissenschaftliche Theoriebildung erlaubt, einen Teil der komplizierten psychologischen Prozesse aufzuklären, die durch sozialen Wandel entstehen und weiteren sozialen Wandel aufgrund der Handlungsfolgen mit beeinflussen.

\section{Literatur}

Bandura, A. (1997). Self-efficacy: The exercise of control. New York: W. H. Freeman.

Berry, J. W. (1990). Psychology of acculturation. In J. J. Berman (Ed.), Nebraska Symposium on Motivation, 1989: Cross-cultural perspectives, Current theory and research in motivation (Vol. 37, pp. 201-234). Lincoln, NE: University of Nebraska Press.

Berry, J. W. \& Kim, U. (1988). Acculturation and mental health. In P. R. Dasen, J. W. Berry \& N. Sartorius (Eds.), Health and cross-cultural psychology (pp. 207. 235). Newbury Park, CA: Sage.

Essau, C. \& Trommsdorff, G. (1993). Kontrollorientierung von Jugendlichen im Kulturvergleich. Zeitschrift für Sozialisationsforschung und Erziehungssoziologie. Schwerpunktheft: Jugend in Kulturvergleich, 4, 311-325.

Gensicke, T. (1996). Von der Transformation zur Integration. Die Ostdeutschen an der Schnittstelle zwischen individueller Anpassung und Vergesellschaftung im neuen Deutschland. Dissertation. Speyer: Hochschule fur Verwaltungswissenschaften.

Hormuth, S. E., Heinz, W. R., Kornadt, H.-J., Sydow, H. \& Trommsdorff, G. (1996). Individuelle Entwicklung, Bildung und Berufsverläufe. Berichte zum sozialen und politischen Wandel in Ostdeutschland der KSPW (Bd. 4). Opladen: Leske + Budrich. 
Lepsius, R. M. (1995). LäBt sich die deutsche Einheit durch die Institutionenordnung herstellen? In Heidelberger Akademie der Wissenschaften (Hrsg.), Jahrbuch der Heidelberger Akademie der Wissenschaften für 1995 (S. 61-66). Heidelberg.

Meulemann, H. (1996). Werte und Wertewandel. Zur Identität einer geteilten und wieder vereinten Nation. Weinheim: Juventa.

Meulemann, H. (1998). Werte und nationale ldentität im vereinten Deutschland. Erklärungsansätze der Umfrageforschung. Opladen: Leske + Budrich.

Montada, L. (1994). Umverteilungen nach der Vereinigung: Über den Bedarf an Psychologie nach dem Beitritt der chemaligen DDR zur Bundesrepublik. In G. Trommsdorff (Hrsg.), Psychologische Aspekte des sozio-politischen Wandels in Ostdeutschland (S, 50-62). Berlin; de Gruyter.

Montada, L. (1997). Gerechtigkeitsansprüche und Ungerechtigkeitserleben in den neuen Bundesländern. In W. R. Heinz \& S. E. Hormuth (Hrsg.), Arbeit und Gerechtigkeit im ostdeutschen Transformationsprozeß (S. 231-274). Opladen: Leske + Budrich.

Moscovici, S. (1985). Perspectives on minority influence. Cambridge: Cambridge University Press.

Nauck, B. \& Joos, M. (1996). Wandel der familiären Lebensverhältnisse von Kindern in Ostdeutschland. In G. Trommsdorff (Hrsg.), Sozialisation und Entwicklung von Kindern vor und nach der Vereinigung. Beiträge zum Bericht „Individuelle Entwicklung, Bildung und Berufsverläufe im Transformationsproze $\beta^{\text {"d }}$ der KSPW (Bd. 4.1, S. 243-298). Opladen: Leske + Budrich.

Rothbaum, F., Weisz, J. R. \& Snyder, S. S. (1982). Changing the world and changing the self: A two-process model of perceived control. Journal of Personality and Social Psychology, 42, 5-37.

Schmitt, M. \& Janetzko, E. (1994). Verantwortlichkeitsüberzeugungen bei Ost- und Westdeutschen. In G. Trommsdorff (Hrsg.), Psychologische Aspekte des soziopolitischen Wandels in Ostdeutschland (S. 169-179). Berlin: de Gruyter.

Seginer, R., Trommsdorf, G. \& Essau, C. (1993). Adolescent control beliefs: Crosscultural variations of primary and secondary orientations. International Journal of Behavioral Development, 16, 243-260.

Trommsdorff, G. (1989). Sozialisation und Werthaltungen im Kulturvergleich. In G. Trommsdorff (Hrsg.), Sozialisation im Kulturvergleich (S. 97-121). Stuttgart: Enke.

Trommsdorff, G. (1994). Future time perspective and control orientation: Social conditions and consequences. In Z. Zaleski (Ed.), Psychology of future orientation (pp. 39-62). Lublin, Poland: TN Kul.

Trommsdorff, G. (1995). Identitätsprozesse im kulturellen Kontext und im sozialen Wandel. In H. Sahner (Hrsg.), Transformationsprozesse in Deutschland (S. 117 148). Opladen: Leske + Budrich.

Trommsdorff, G. (in Druck a). Social change and individual development in East Germany: A methodological critique. In R. K. Silbereisen \& A. von Eye (Eds.), Growing up in times of social change. Berlin: de Gruyter.

Trommsdorff, G. (in Druck b). Effects of social change and individual development: The role of social and personal factors, and the timing of events. In L. Crocket $\&$ R. K. Silbereisen (Eds.), Negotiating adolescence in times of social change. Cambridge: Cambridge University Press. 
Trommsdorff, G. (in Druck c). Social change and individual development: The role of subjective beliefs. In R. K. Silbereisen \& J. Bynner (Eds.), Adversity and challenge in life in the new Germany and England. Hampshire, England: The MacMillan Press Ltd.

Trommsdorff, G. \& Essau, C. A. (in Druck). Japanese and German adolescents' control orientation: A cross-cultural study. In G. Trommsdorff, W. Friedlmeier \& H.J. Kornadt (Eds.), Japan in transition - A comparative view on social and psychological aspects. Lengerich: Pabst Science Publishers. 